\title{
Experiencia de hipotermia terapéutica en una unidad de cuidados intensivos
}

\author{
Gilberto Adrián Gasca-lópez ${ }^{1}$, Alfredo Arellano-Ramírez ${ }^{1}$, Rodolfo Pinto-Almazán², \\ Evelyn Galindo-Oseguera ${ }^{3}$ y Lucero Durán-Santana ${ }^{3}$ \\ ${ }^{1}$ Unidad de Cuidados Intensivos; ${ }^{2}$ Investigador en ciencias médicas C: ${ }^{3}$ Unidad de Investigación. Hospital Regional de Alta Especialidad de Ixtapaluca, Ixtapaluca, México
}

\section{RESUMEN}

Introducción: La hipotermia terapéutica (HT) está indicada para la protección cerebral en casos de riesgo de lesión; sin embargo, por sus efectos electrolíticos su uso es controversial. Objetivo: Determinar las variaciones electrolíticas y metabólicas de la HT en sus distintas fases, las complicaciones que generan y su relación con la mortalidad en la Unidad de Cuidados Intensivos (UCI) de adultos del Hospital Regional de Alta Especialidad de Ixtapaluca (HRAEI). Métodos: Estudio retrospectivo, observacional y descriptivo, con método no paramétrico con la $t$ de Student apareada, de los pacientes sometidos a HT durante los meses de abril a septiembre de 2017. Resultados: Se sometieron a HT 9 individuos, alcanzando la temperatura ideal $\left(33^{\circ} \mathrm{C}\right)$ a las $3 \mathrm{~h}$. El potasio $(\mathrm{K})$ presentó valores normales en el $66.6 \%$ de los pacientes con el aporte del mismo. En el $77.7 \%$ de los se presentó hiperglucemia en las fases de inducción y mantenimiento, y glucemia normal en el recalentamiento (66.6\%). Discusión: Otros estudios han evaluado los efectos de la HT sin estandarizar aún la temperatura ideal para evitar complicaciones. En este estudio los pacientes no presentaron alteraciones similares a lo descrito en la literatura médica. La HT mejora el edema cerebral; sin embargo, por sus efectos adversos, la monitorización estrecha de electrólitos es fundamental y su tratamiento específico impacta en el pronóstico y disminuye la mortalidad.

Palabras clave: Hipotermia inducida. Desequilibrio hidroelectrolítico. Traumatismo craneoencefálico.

\section{ABSTRACT}

Introduction: Therapeutic hypothermia $(\mathrm{TH})$ is indicated for brain protection; however, due to its electrolytic effects, its use is controversial. Objective: We analyzed the electrolyte and metabolic variations during $\mathrm{TH}$ in its different phases, the complications generated by these variations, and their relationship with mortality in an adult intensive care unit. Methods: We conducted a retrospective, observational and descriptive study of patients subjected to TH from April through September 2017 at the High-Specialty Regional Hospital of Ixtapaluca, Mexico. Data were analyzed using the nonparametric method with paired Student's $t$ test. Results: During the study period, 9 individuals underwent

\section{Correspondencia:}

*Gilberto Adrián Gasca-López

Unidad de Cuidados Intensivos

Hospital Regional de Alta Especialidad de Ixtapaluca

Carretera Federal México-Puebla, km 34.6

Date of reception: 28-04-2018

C.P. 56530, Pueblo de Zoquiapan, Ixtapaluca, México

Date of acceptance: 10-10-2018

E-mail: gadriangasca1@yahoo.com.mx

DOI: 10.24875/HMCM.18000125 
$\mathrm{HT}$, reaching the ideal temperature $\left(33^{\circ} \mathrm{C}\right)$ at 3 hours. Potassium values were normal in $66.6 \%$ with its supplementation; $77.7 \%$ had hyperglycemia during the induction and maintenance phases, returning to normal glycemia in the re-warming period (66.6\%). Discussion: Other studies have evaluated the effects of HT, without standardizing for the ideal temperature to prevent complications. Although HT improved cerebral edema, because of its adverse effects, closely monitoring of the electrolyte balance is fundamental and its specific treatment improves the prognosis, decreasing mortality. (Hosp Med Clin Manag. 2018;11:178-85)

Corresponding author: Gilberto Adrián Gasca-Lópezr, gadriangasca1@yahoo.com.mx

Key words: Induced hypothermia. Fluid and electrolyte Imbalance. Traumatic brain injury.

\section{INTRODUCCIÓN}

La HT consiste en la reducción controlada de la temperatura central de un paciente en un intento de proteger un órgano en riesgo de lesión. El uso de la HT se remonta al antiguo Egipto y China, donde el frío era usado como tratamiento del dolor. En 1940, el neurocirujano T. Fay lo utilizó para trauma craneoencefálico ${ }^{1}$. Desde entonces, se ha utilizado por sus grandes beneficios, ya que mejora la supervivencia y los resultados neurológicos por la protección cerebral en casos de paro cardiorrespiratorio y encefalopatía hipóxico-isquémica ${ }^{2,3}$. Además, se ha observado que es eficaz para disminuir la presión intracraneal ${ }^{4}$. Sin embargo, debido a sus efectos a nivel sistémico y celular puede desencadenar trombosis, modulación inmune, lesiones térmicas, arritmias, infecciones secundarias, temblores, pancreatitis y desequilibrio hidroelectrolítico ${ }^{5}$. En cuanto al desequilibrio hidroelectrolítico, éste se debe a la actividad de la fosfolipasa A2, la cual desencadena hidrólisis de las paredes celulares presentándose fuga de iones, principalmente de $\mathrm{K}$, lo que puede incrementar la mortalidad $^{3,5,6}$.

El objetivo de este estudio es mostrar la experiencia de las variaciones electrolíticas y metabólicas inducidas por la HT durante sus distintas fases, las complicaciones que generan y su relación con la mortalidad en la UCl de adultos del HRAEI.

\section{MATERIAL Y MÉTODOS}

Se llevó a cabo un estudio retrospectivo, observacional y descriptivo en el que se formó una base de datos general con el servidor Excel durante los meses de abril a septiembre de 2017. La información se recabó de los expedientes clínicos electrónicos del HRAEI. Se realizó el análisis estadístico empleando el programa
Graphpad Prisma 6.0 y efectuando una prueba $t$ de Student apareada para los electrólitos séricos (sodio $[\mathrm{Na}], \mathrm{K}$, cloro [Cl], fósforo [P], magnesio [Mg] y calcio [Ca] corregidos] y glucosa durante el ingreso, las fases de HT (inducción, mantenimiento y recalentamiento) y al egreso. Se analizaron los estudios de gabinete antes y después en los pacientes sometidos a HT en la UCl.

\section{Técnica de hipotermia terapéutica}

Como medidas generales deben considerarse los siguientes aspectos al emplear esta técnica terapéutica:

- La HT debe iniciarse lo más rápido posible; en nuestro caso, el método de enfriamiento que se utilizó fue mediante el sistema de almohadillas de gel (Pads de gel) de Arctic Sun.

- Para iniciar el procedimiento es necesario verificar previamente el estado de sedación con el fin de evitar calosfríos durante el procedimiento, y debe colocarse un sistema de monitoreo integral que incluya: frecuencia cardíaca, presión arterial media, presión venosa central, ventilación mecánica, reposición de electrólitos y monitoreo electroencefalográfico.

- Se debe tomar hemocultivo a las $12 \mathrm{~h}$ posteriores al inicio de la HT y revisar cada $2 \mathrm{~h}$ el sitio donde se colocaron los Pads de gel, por riesgo de quemaduras causadas por el sistema de enfriamiento.

Las fases de esta terapia se realizan de la siguiente forma:

- Inducción: con soluciones frías $\left(4^{\circ} \mathrm{C}\right)$, calculando un volumen de líquidos de $30 \mathrm{ml} / \mathrm{kg}$ para lograr llegar a la meta de enfriamiento $\left(33-34^{\circ} \mathrm{C}\right)$ lo más rápidamente posible, preferentemente en la primera hora. 
- Mantenimiento: el sistema Arctic Sun posee parámetros para cada fase, por lo que sólo se mantiene en vigilancia y se corrigen las alteraciones hidroelectrolíticas que se presenten durante 24-72 h.

- Recalentamiento: se realiza con un aumento de temperatura de $0.25-0.5^{\circ} \mathrm{C}$ por hora hasta llegar a $36^{\circ} \mathrm{C}$, lo cual se programa en el sistema Arctic Sun. Cabe mencionar que $4 \mathrm{~h}$ antes del inicio de esta fase se deberá suspender la reposición de electrólitos por el riesgo de presentar hipercalemia.

- Normotermia: durante al menos $24 \mathrm{~h}$ se debe mantener la temperatura de $36^{\circ} \mathrm{C}$ a fin de evitar la hipertermia o la hipotermia.

\section{RESULTADOS}

En la UCI del HRAEl en los meses de abril a septiembre de 2017 se sometieron a HT 9 individuos -1 mujer $(11.1 \%)$ y 8 hombres $(88.8 \%)-$, con un rango de edad de 18 a 72 años; la edad media fue de 30.2 años, mediana de 24 y desviación estándar de 17. Con respecto a los diagnósticos de ingreso a la $\mathrm{UCl}$, éstos fueron traumatismo craneoencefálico severo (88.8\%) y síndrome posparada cardíaca (11.1\%).

La media de estancia en la $\mathrm{UCl}$ fue de $8.1 \pm 3$ días (mínimo de 5 días y máximo de 15 días).

El tiempo para alcanzar la temperatura en la fase de inducción fue de $3 \mathrm{~h}$ para el $55.5 \%$ de los pacientes (Fig. 1). El promedio de tiempo para las fases de HT fue de $4 \mathrm{~h}$ para la de inducción, $24 \mathrm{~h}$ para la de mantenimiento, $16 \mathrm{~h}$ para la de recalentamiento y $24 \mathrm{~h}$ para la normotermia.

Los electrólitos que se valoraron fueron $\mathrm{Na}, \mathrm{K}, \mathrm{Cl}, \mathrm{P}, \mathrm{Mg}$ y Ca. El $\mathrm{Na}$ al ingreso se encontró normal en el $88.8 \%$ de los pacientes (parámetro de $135-145 \mathrm{mEq} / \mathrm{l}$ ) y el $11.1 \%$ presentó hipernatremia (máximo de $147 \mathrm{mEg} / \mathrm{l}$ ). En la fase de inducción, el 55.5\% tuvo valores de Na normales y el 44.4\% hipernatremia (máximo de $153 \mathrm{mEq} / \mathrm{l}$ ). En la fase de mantenimiento, el $77.7 \%$ presentó hipernatremia (máximo de $167 \mathrm{mEq} / \mathrm{ll}$ y el resto (22.2\%) valores normales. En la fase de recalentamiento, el $66.6 \%$ presentó hipernatremia (máximo de $157 \mathrm{mEq} / \mathrm{l}$ ) y el resto estuvo dentro de los parámetros normales (33.3\%); sin embargo, en la normotermia al término de la HT los niveles de Na no cambiaron.

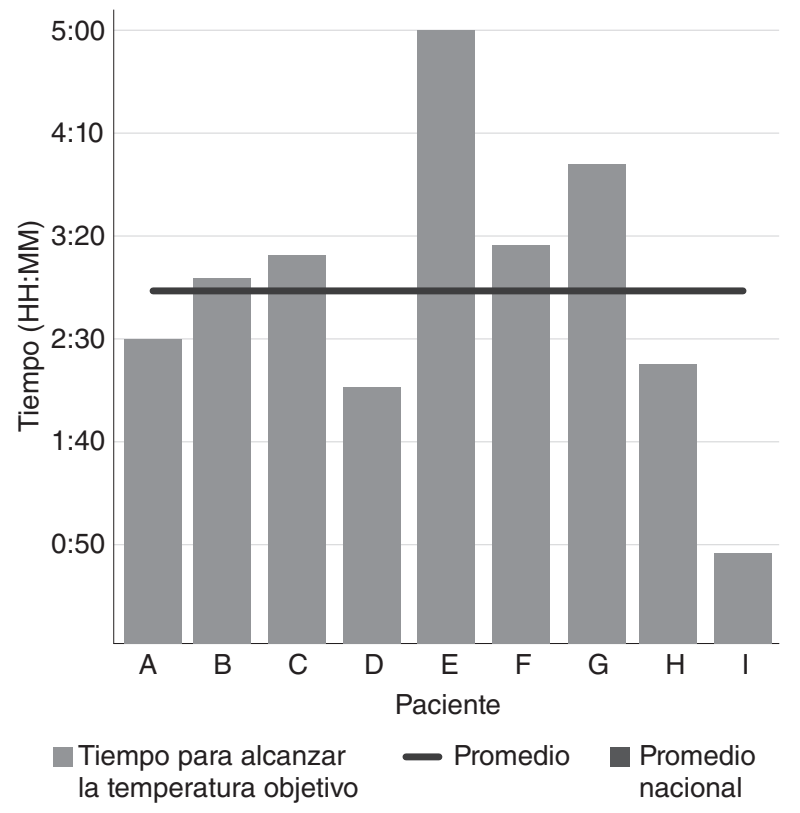

Figura 1. Tiempo para alcanzar la temperatura objetivo en la hipotermia terapéutica de 34 grados centígrados que corresponden en 3 horas promedio.

En cuanto al K, en la fase de inducción en el $66.6 \%$ fue normal y sólo el $22.2 \%$ presentó hipocalemia (mínimo de $2.8 \mathrm{mEq} / \mathrm{l})$. Durante la fase de mantenimiento, el $88.8 \%$ mantuvo cifras normales y el $11.1 \%$ presentó hipercalemia de $5.7 \mathrm{mEq} / \mathrm{l}$. Por último, en la fase de recalentamiento el $66.6 \%$ mantuvo sus cifras normales, sólo un $11.1 \%$ presentó hipercalemia y otro $11.1 \%$ hipocalemia, de 6.9 y $3.2 \mathrm{mEq} / \mathrm{l}$, respectivamente.

Sobre la reposición de K para los pacientes con hipocalemia, no se administró en la fase de inducción, pero sí en la fase de mantenimiento al $88.8 \%$ de los casos, y al $66.6 \%$ en la fase de recalentamiento, con vigilancia estrecha para mantener cifras de 4-5 mEq/l. Sin embargo, durante esta última el aporte se suspendió a los $34{ }^{\circ} \mathrm{C}$ para evitar hipercalemia, ya que es la fase en la que se reporta en la literatura médica el mayor número de complicaciones por los tratamientos adyuvantes. Por lo tanto, la relación que existe entre los niveles de $\mathrm{K}$ al inicio y al final de la HT se mantuvieron estables (Figs. 2 A y B).

$\mathrm{El} \mathrm{Cl}$ al ingreso no tuvo alteración, pero en las fases de inducción, mantenimiento y recalentamiento presentaron hipercloremia el 66.6\% (máximo de $122 \mathrm{mEq} / \mathrm{l}$ ), el 88.8\% (máximo de $146 \mathrm{mEq} / \mathrm{l}$ ) y el 66.6\% (máximo de $129 \mathrm{mEq} / \mathrm{l}$ ), respectivamente. Sin embargo, en la normotermia, el 


\section{A. Niveles de Na sérico durante la HT}

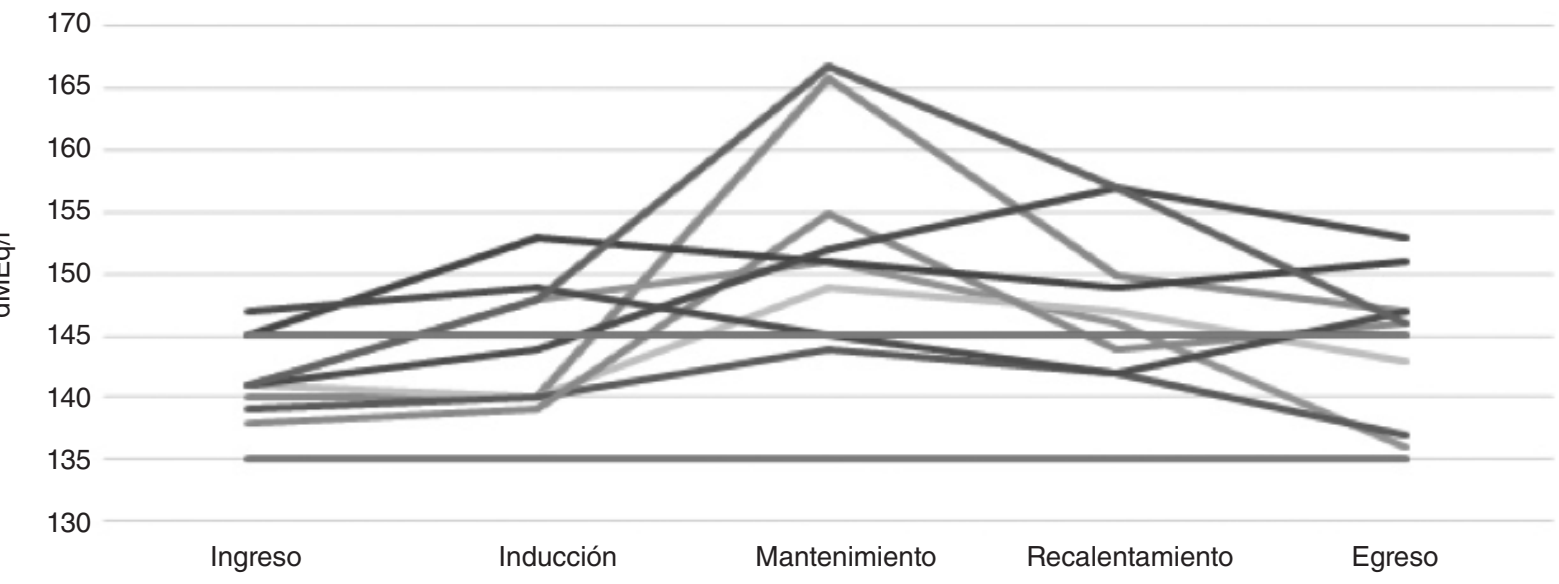

B. Biveles de $\mathrm{K}$ sérico durante la HT

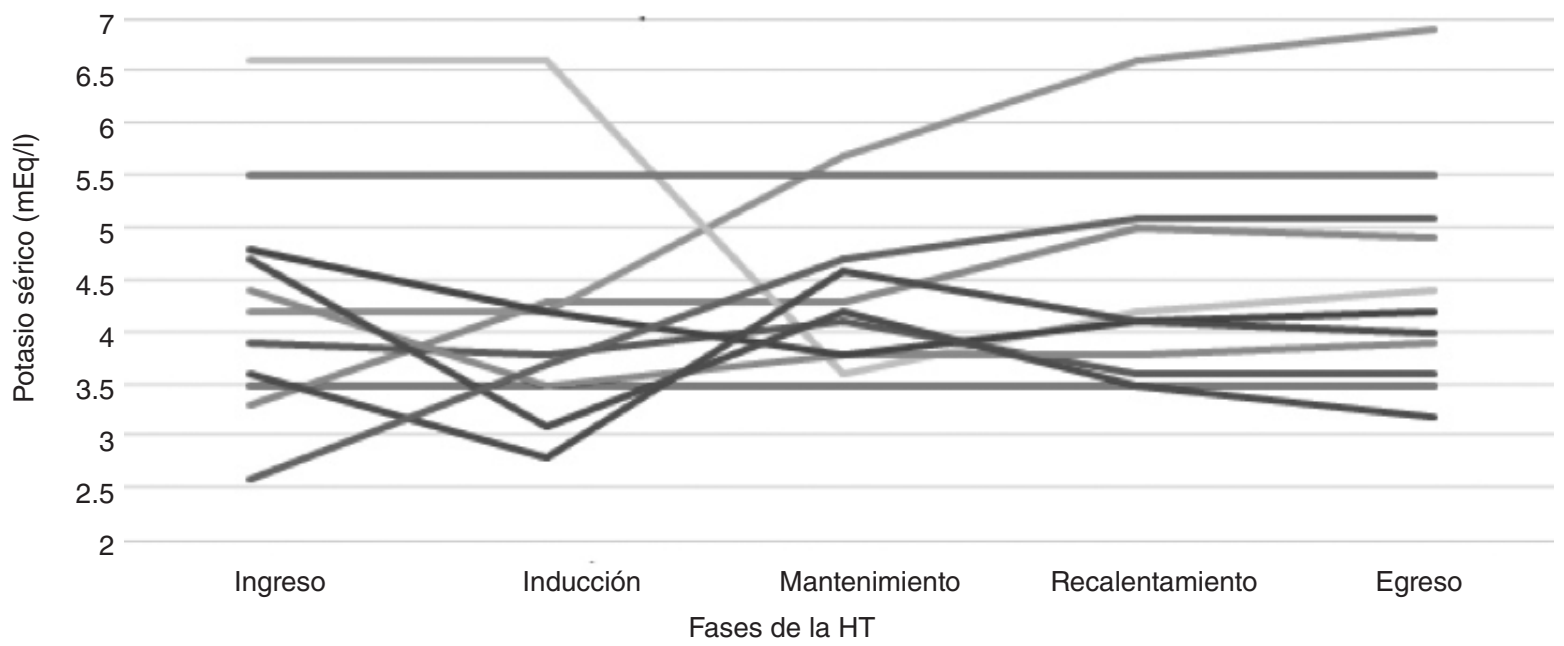

C. Niveles de glucosa sérica durante la HT

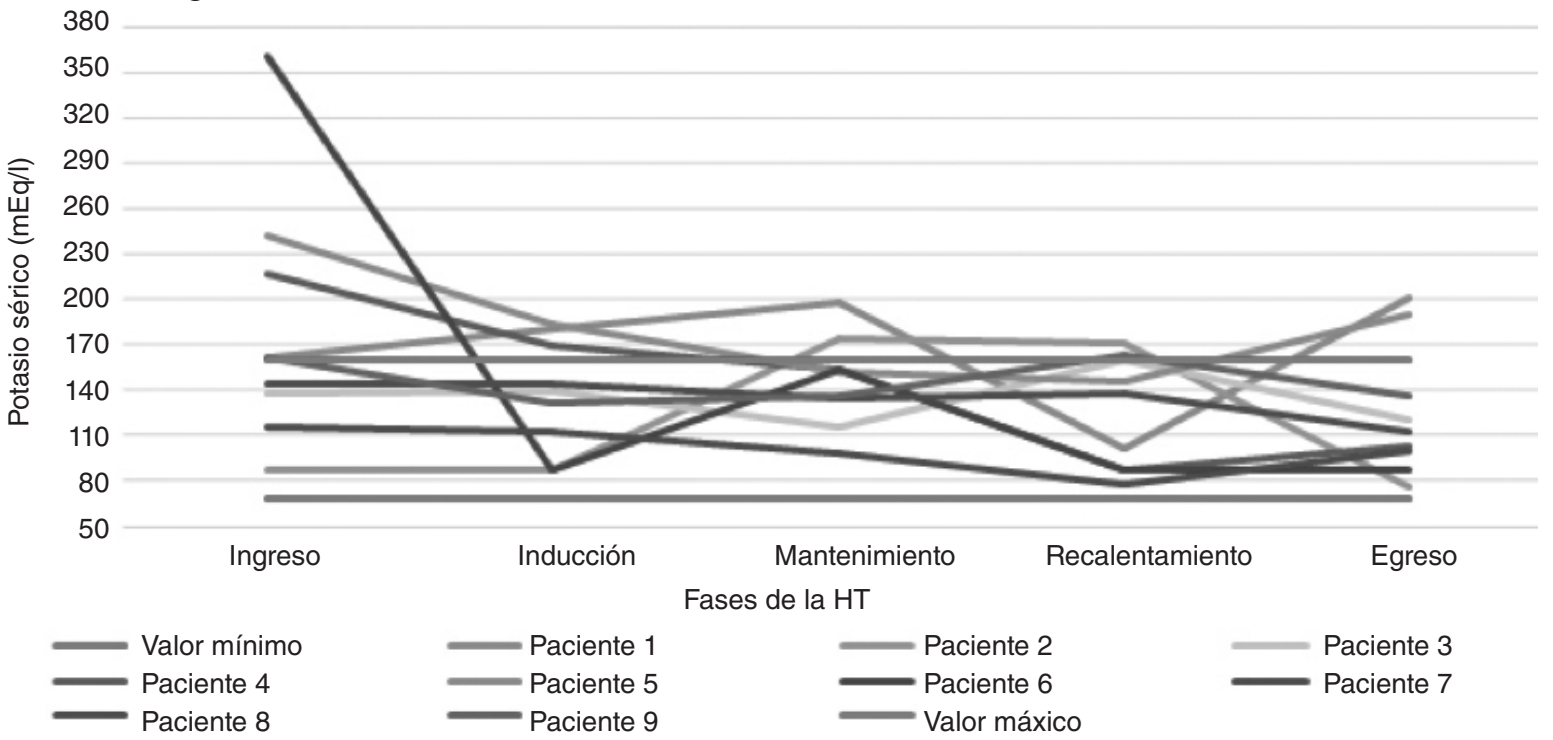

Figura 2. Niveles de $\mathrm{Na}, \mathrm{K}$ y glucosa en las diferentes fases de la HT. 
$55.5 \%$ pudo mantener sus niveles normales, sólo el 33.3\% se mantuvo con hipercloremia y un $11.1 \%$ presentó hipocloremia (Fig. 3 A).

Con respecto al $\mathrm{P}$, al inicio el $88.8 \%$ tuvo valores normales (2.5-5 mg/dl) y el $11.1 \%$ presentó hipofosfatemia (mínimo de $1.9 \mathrm{mg} / \mathrm{dl}$ ). En la fase de inducción la misma cantidad mantuvo sus niveles normales, sólo que ahora el 11.1\% presentó hiperfosfatemia (máximo de $6.2 \mathrm{mg} / \mathrm{dll}$ ); y durante la fase de mantenimiento el $22.2 \%$ continuó con hiperfosfatemia (máximo de $8.2 \mathrm{mg} / \mathrm{dl}$ ) y el $11.1 \%$ presentó hipofosfatemia (mínimo de $1.4 \mathrm{mg} / \mathrm{dl}$ ); el $66.6 \%$ restante se mantuvo en cifras normales. En la fase de recalentamiento presentaron hipofosfatemia el 22.2\% (mínimo de $1 \mathrm{mg} / \mathrm{dl}$ ), en el resto fue normal. El $88.8 \%$ mantuvo sus cifras normales al término de la HT (Fig. 3 B).

En cuanto al Mg, sólo un $11.1 \%$ presentó hipomagnesemia durante todas las fases de HT (inducción, mantenimiento y recalentamiento), con unos mínimos de 1.1, 1.1 y $1.2 \mathrm{mg} / \mathrm{dl}$, respectivamente, siendo normal en todos los individuos al ingreso y al llegar a la normotermia al término de la terapia hipotérmica (Fig. 3 C).

Con respecto al $\mathrm{Ca}$, el $11.1 \%$ presentó hipocalcemia al inicio (mínimo de $8.1 \mathrm{mg} / \mathrm{dl}$ ), en la fase de inducción (mínimo de $7.8 \mathrm{mg} / \mathrm{dl}$ ), en la fase de mantenimiento el $22.2 \%$ (mínimo de $8.4 \mathrm{mg} / \mathrm{dl}$ ) y en la de recalentamiento nuevamente el $11.1 \%$ (mínimo de $8.4 \mathrm{mg} / \mathrm{dl}$ ). En el resto de pacientes estuvo dentro de los parámetros normales (Fig. 3 D).

Metabólicamente, la glucosa sérica es otra molécula íntimamente afectada durante la HT. En la fase de inducción, el $66.6 \%$ de los pacientes mantuvo sus niveles normales para el paciente crítico (70-160 mg/dl) y sólo un 33.3\% presentó hiperglucemia (máximo de $185 \mathrm{mg} / \mathrm{dl}$ ). Ahora bien, en la fase de mantenimiento, el $77.7 \%$ mantuvo glucemias normales y el $33.3 \%$ hiperglucemia (máximo de $200 \mathrm{mg} / \mathrm{dl}$ ). En la fase de recalentamiento, el $66.6 \%$ mantuvo cifras normales e hiperglucemia en el 33.3\% (máximo de 172 mg/dl).

Ante las descompensaciones metabólicas en la glucosa, se inició manejo con insulina de acción rápida en el $22.2 \%$ durante la fase de inducción, en el $66.6 \%$ durante la fase de mantenimiento (a pesar de encontrarse dentro de los parámetros normales) y fue suspendida en la fase de recalentamiento por riesgo de hipoglucemia, por lo que al final de la HT la mayoría de los individuos (88.8\%) presentó cifras dentro de los parámetros normales (Fig. $3 \mathrm{C}$ ).

Conforme al egreso, en el $77.7 \%$ fue por mejoría por disminución del edema cerebral y evolución favorable del estado neurológico, y en el 22.2\% por defunción secundaria a traumatismo craneoencefálico severo por daño neurológico irreversible. Por tanto, la mortalidad en estos casos fue baja, aun con las variaciones electrolíticas o metabólicas que generó la HT.

\section{DISCUSIÓN}

Reportes previos han demostrado que la hipotermia aumenta la estabilidad de la membrana celular; sin embargo, el enfriamiento parece engendrar una etapa susceptible a procesos de arritmia, lo cual es potencialmente agravado por una isquemia coexistente $o$ una lesión por reperfusión en las membranas celulares ${ }^{1,6-9}$.

Se han efectuado múltiples estudios para determinar los beneficios y los efectos adversos producidos por la HT; sin embargo, aún no se ha logrado estandarizar la temperatura ni la velocidad ideal para evitarlos, por lo que existe discrepancia en su empleo.

En la revisión de Moore, sw hace referencia al estudio de Clifton, donde el tiempo para fijar la temperatura fue de $8.4 \mathrm{~h}$, un subgrupo de pacientes al llegar al hospital tuvo resultados neurológicos a largo plazo significativamente mejores en comparación con los pacientes normotérmicos, lo que puede apoyar el efecto beneficioso propuesto de la $\mathrm{HT}^{3}$.

En la Guía Canadiense para el Uso del Manejo de la HT después del Paro Cardiorrespiratorio (una declaración conjunta de la Sociedad Canadiense de Cuidados Críticos, la Sociedad Canadiense de Cuidados Neurocríticos y los Grupos de Prueba de los Cuidados Críticos Canadienses) de 2016, se recomienda que los niveles de $\mathrm{K}$ se mantengan por encima de $3.0 \mathrm{mmol}$ durante la fase de inducción, con su respectivo aporte con vigilancia estrecha ${ }^{6,10}$, sobre todo en la fase de recalentamiento, en la que surge un efecto de rebote con hipercalemia, ya que genera el mayor número de complicaciones.

Busea, et al. concluyeron en su revisión que la fase final de la hipotermia puede conducir a hipercalemia, lo que es un indicador de mal pronóstico por el estrés metabólico 


\section{A. Niveles de Na sérico durante la HT}

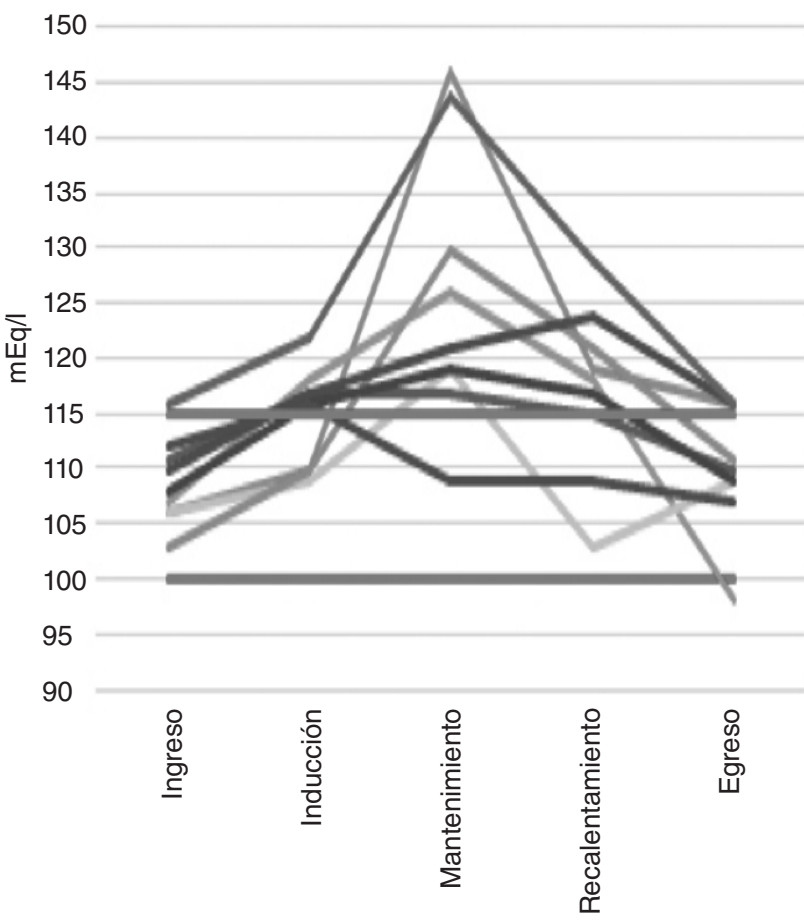

C. Niveles de magnesio sérico durante la HT

3.0
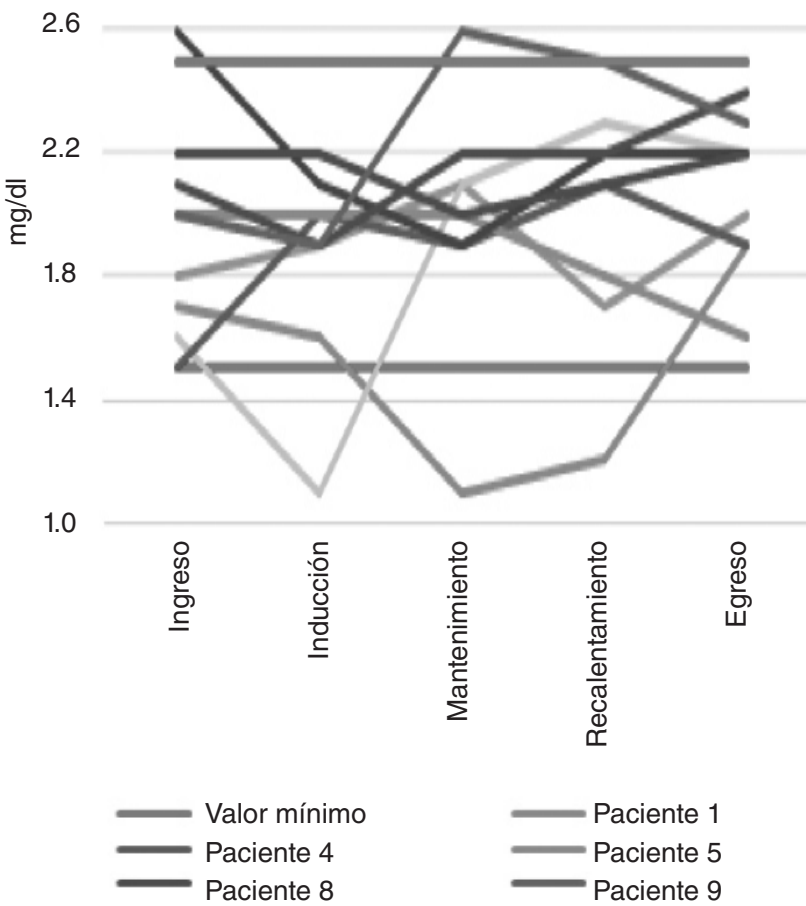

\section{B. Niveles de P sérico durante la HT}

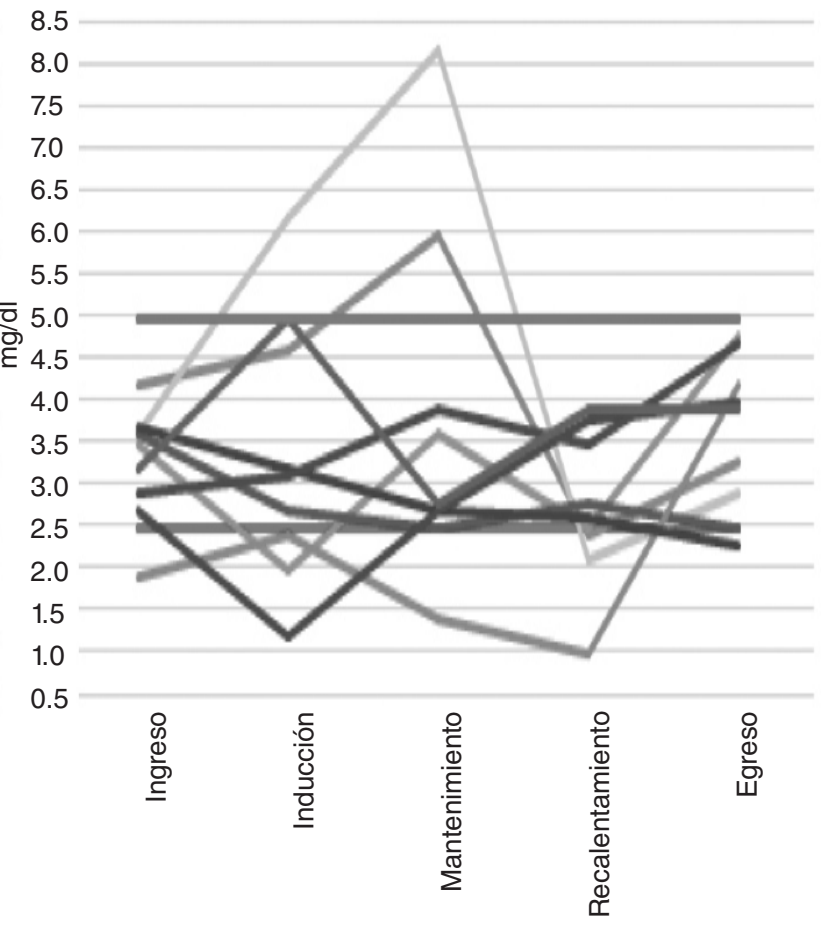

D. Niveles de calcio sérico durante la HT 11.0

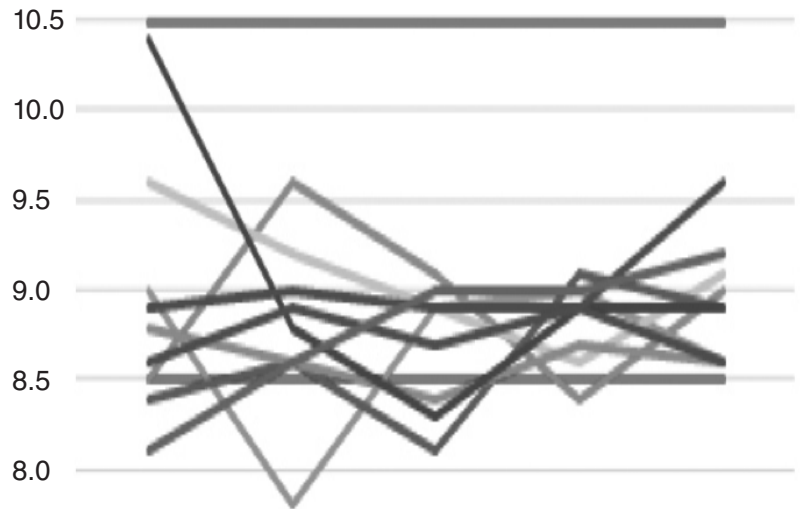

7.5

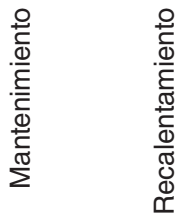

Paciente 3

Paciente 7

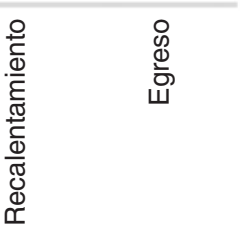

Paciente 2

Paciente 6

Valor máxico

Figura 3. Electrólitos séricos en las diferentes fases de la HT. 
generado y el daño celular irreversible, por lo que consideran que este electrólito es el más importante ${ }^{6,11,12}$; sin embargo, en este estudio sólo un individuo presentó hipercalemia y el $88.8 \%$ mantuvo sus niveles normales, por lo que las condiciones de gravedad de su situación fueron las que intervinieron en su pronóstico, y no la HT.

En el estudio realizado por Mirzoyev, et al., se determinó que la hipocalemia severa $(\leq 3 \mathrm{mmol} / \mathrm{l})$ en la fase de inducción puede considerarse como un valor predictivo para taquicardia ventricular ( $p=0.001)$, pero la hipercalemia no ${ }^{13}$. En contraste con el presente estudio, durante la fase de inducción el $66.6 \%$ mantuvo sus niveles normales, pero los que presentaron hipocalemia no tuvieron ninguna complicación, lo que no afectó a la mortalidad. Ahora bien, los autores también refieren que durante las fases de mantenimiento y recalentamiento los niveles pueden mantenerse estables con aporte de K, como sucedió en el $64 \%$ de los casos que reportaron, mientras que en el presente estudio fue en el $88.8 \%$, lo que nos sugiere que el aporte de $\mathrm{K}$ está indicado en estas fases, sólo que requiere una vigilancia estrecha y la suspensión de éste a los $34^{\circ} \mathrm{C}$ para evitar como efecto la Hiperkalemia. Además, se demuestra que los niveles de Mg disminuyeron en 3 pacientes, por lo que requirieron aporte sin tener relación alguna con taquicardia ventricular. Lo que en esta experiencia se demuestra es que el efecto es posible, por el $11.1 \%$ que sí presentaron esto; sin embargo, no fue significativo para generar complicación alguna o que afectara en la mortalidad

En un ensayo realizado por el Hypothermia After Cardiac Arrest Study Group ${ }^{15}$ se evaluaron 275 individuos, de los cuales 137 ingresaron a HT durante $24 \mathrm{~h}$ con mejoría neurológica del 55\% ( $p=0.009)$ y disminución de la mortalidad del $41 \%(p=0.002)$ a los 6 meses. Esto concuerda con el estudio de Bernard, et al. ${ }^{16}$, quienes expusieron a 43 de 77 pacientes a HT por $24 \mathrm{~h}$ y encontraron una recuperación neurológica del $49 \%$, con respecto al $26 \%$ de los individuos no sometidos a $\mathrm{HT}$, siendo esto favorable, ya que en el $77.7 \%$ mejoró su estado neurológico a pesar de la gravedad de la situación. Igualmente concuerda con lo referido en las guías de la American Heart Association (AHA) de 2010. de 2010, las cuales recomiendan fuertemente la HT por ser neuroprotectora en los casos de hipoxia cerebral secundaria a paro cardíaco, disminuyendo el consumo de oxígeno y glucosa en las primeras fases de daño cerebral ${ }^{15-17}$
Boodhwani, et al. realizaron 2 ensayos aleatorios en pacientes con bypass cardiopulmonar, el primero con 233 pacientes y el segundo con 267, sin recalentamiento, en los cuales se evaluaron la relación de HT leve con los niveles de creatinina sérica y la depuración de creatinina. Se observó que los pacientes recalentados a $37^{\circ} \mathrm{C}$ presentaban una mayor incidencia de lesión renal y mayor tiempo de estancia hospitalaria, a diferencia de los pacientes con $34{ }^{\circ} \mathrm{C}(17$ vs. $9 \% ; p=0.07$ ). Se concluyó que el recalentamiento en estos pacientes se relacionó con aumento de lesión renal aguda, por lo que debe evitarse ${ }^{3,18}$.

En contraste, en nuestro estudio el $\mathrm{K}$ también fue el electrólito mayormente afectado, presentándose hipocalemia durante la fase de inducción e hipercalemia en la fase de recalentamiento. A diferencia del estudio Clifton citado en la revisión de Moore, que refiere hipocalemia severa, en el presente estudio también se correlaciono, lo cual probablemente se relacione con el tiempo en que se alcanzó la temperatura ideal, ya que en este estudio fue de $3 \mathrm{~h}$ variando en las fases de HT, además de que se presentó hipercalemia en un porcentaje menor, lo que sugiere un adecuado control del aporte del mismo y se relaciona con lo recomendado por la Guía Canadiense para el Uso del Manejo de la HT después del Paro Cardiorrespiratorio. Sin embargo, también se presentó hipernatremia en la mayoría de los pacientes durante la HT, así como hipercloremia, esta última normalizándose al egreso. Los niveles de fosfato, $\mathrm{Ca}$ y $\mathrm{Mg}$ en los pacientes del presente estudio se encontraron en la mayoría dentro de los límites normales. Este dato no se ha descrito ampliamente en otros estudios, por lo que resulta difícil realizar una comparación, pero sus alteraciones no fueron tan marcadas como para comprometer el pronóstico de los pacientes.

En cuanto a la mortalidad, se encontró disminuida, relacionándose al adecuado monitoreo, reposición de electrólitos y control glucémico, además del corto periodo para alcanzar la temperatura ideal y el tiempo en que se mantuvo cada una de las fases de HT, ya que el $77.7 \%$ de los pacientes fue egresado por mejoría, mientras que el 22.2\% por defunción secundaria a traumatismo craneoencefálico severo. En estos últimos fue la gravedad de su padecimiento lo que intervino en su deceso, y no precisamente la $\mathrm{HT}$, ya que los valores de electrólitos se encontraban en su mayoría dentro de los parámetros normales. Además, hubo una mejoría marcada en cuanto al estado neurológico que se reflejó en los estudios 
de imagen, los cuales mostraron una disminución significativa del edema cerebral, a excepción de los decesos, los cuales mostraron un daño neurológico irreversible, aun con todas las medidas antiedema cerebral.

En los últimos años se ha identificado que la HT actúa manteniendo la integridad del citoesqueleto celular y disminuye el intercambio iónico, creando estabilidad en la membrana. Por este principio la atención se ha centrado en la forma en que se manifiestan las alteraciones hidroelectrolíticas durante el empleo de esta técnica terapéutica.

\section{CONCLUSIÓN}

La HT implica la reducción controlada de la temperatura con vigilancia estrecha del equilibrio hidroelectrolítico y metabólico. Sin embargo, al llegar a las $3 \mathrm{~h}$ a la temperatura ideal, con $24 \mathrm{~h}$ de mantenimiento, $16 \mathrm{~h}$ de recalentamiento y la suspensión de aporte de $\mathrm{K}$ e insulina a los $34^{\circ} \mathrm{C}$ de la última fase, se mostró el control constante en la mayoría de los individuos, lo que disminuye el riesgo de mortalidad en los pacientes con traumatismo craneoencefálico severo. Debido al reducido número de individuos sometidos a HT, los resultados de este estudio deben tomarse con reserva.

\section{BIBLIOGRAFÍA}

1. Rojas MG. Aplicación de un protocolo de hipotermia controlada a pacientes adultos posparo cardiaco en el Hospital el Tunal E.S.E. para evaluar la mortalidad y funcionalidad neurológica a los 28 días. Universidad Nacional de Colombia; 2014.
2. Akrivi Manola, Giovanni G. Geronilla, Kamala Ramya Kallur, Hanna Slim, Justin Lundbye. The impact of therapeutic hypothermia on serum potassium. Journal of the American College of Cardiology. 2012;59(13):E62. Disponible en http://www.onlinejacc.org/content/59/13 Supplement/E62

3. Moore AE, Nichol AD, Bernard SA, Bellomo R. Therapeutic hypothermia: benefits, mechanisms and potential clinical applications in neurological, cardiac and kidney injury. Injury. 2011;42(9):843-54.

4. Dunkley S, McLeod A. Terapeutic hypothermia in patients following traumatic brain injury: a systematic review. Nurs Crit Care. 2017;22(3):150-60.

5. National Institute for Health and Clinical Excellence (NICE) [Internet]. March 2011. Therapeutic hypothermia following cardiac arrest, Interventional procedures guidance [IPG386]; [Consultado 18 Diciembre del 2017]. Disponible en https://www.nice.org.uk/guidance/ipg386/evidence

6. Busea S, Blancher M, Viglino D, Pasquier M, Maignan M, Bouzat $P$, et al. The impact of hypothermia on serum potassium concentration: a systematic review. Resuscitation. 2017;118:35-42. Disponible en https://www.ncbi. nlm.nih.gov/pubmed/28689048

7. Shah N, Garg J, Agarwal V, Mehta K, Jacobs J, Patel N, et al. Stent thrombosis is not increased in cardiac arrest patients undergoing therapeutic hypothermia: an analysis of 15,079 procedures. JAAC. 2015;65(10S):1177.

8. Borges N, Holmes B, Young M, Song Y, McPherson J. Neurologic outcomes with therapeutic hypothermia post-cardiac arrest: developing a prognostication model. JACC. 2015;65(10S):1209.

9. Arrich J, Holzer M, Havel C, Müllner M, Herkner H. Hypothermia for neuroprotection in adults after cardiopulmonar resuscitation. Cochrane Database Syst Rev. 2016;2:CD004128.

10. Howes D, Gray SH, Brooks SC, Boyd JG, Djogovic D, Golan E, et al. Canadian Guidelines for the use of targeted temperature management (therapeutic hypothermia) after cardiac arrest: a joint statement from The Canadian Critical Care Society (CCCS), Canadian Neurocritical Care Society (CNCS), and the Canadian Critical Care Trials Group (CCCTG). Resuscitation. 2016;98:48-63.

11. Alderson P, Gadkary C, Signorini DF. Therapeutic hypothermia for head injury. Cochrane Database Syst Rev. 2004;(4):CD001048.

12. Rim HT, Ahn JH, Kim JH, Oh JK, Song JH, Chang IB. Therapeutic hypothermia for increased intracranial pressure afther descompressive craniectomy: a single center experience. Korean J Neurotrauma. 2016;12(2):55-60.

13. Mirzoyev SA, McLeod CJ, Bunch TJ, Bell MR, White RD. Hypokalemia during the cooling phase of therapeutic hypothermia and its impact on arrhythmogenesis. Resuscitation. 2010;81(12):1632-6.

14. Zydlewski AW, Hasbargen JA. Hypothermia-induced hypokalemia. Mil Med. 1998;163(10):719-21.

15. Hypothermia after Cardiac Arrest Study Group. Mild therapeutic hypothermia to improve the neurologic outcome after cardiac arrest. N Engl J Med. 2002;346(8):549-56.

16. Bernard SA, Gray TW, Buist MD, Jones BM, Silvester W, Gutteridge G, et al. Treatment of comatose survivors of out-of-hospital cardiac arrest with induced hypothermia. N Engl J Med. 2002;346(8):557-63.

17. Peberdy MA, Callaway CW, Neumar RW, Geocadin RG, Zimmerman JL, Donnino M, et al. Part 9: post-cardiac arrest care: 2010 American Heart Association Guidelines for Cardiopulmonary Resuscitation and Emergency Cardiovascular Care. Circulation. 2010;122(18 Suppl 3):S768-86.

18. Boodhwani M, Rubens FD, Wozny D, Nathan HJ. Effects of mild hypothermia and rewarming on renal function after coronary artery bypass grafting. Ann Thorac Surg. 2009;87(2):489-95. 